\title{
A Case Report of Classical eight-and-a-Half Syndrome and Etiology
}

\author{
Duo-Duo Fan ${ }^{1,2}$, Zhe Wang ${ }^{1}$, Wen-Song Mu' ${ }^{2}$, Ming-Ou Lu ${ }^{{ }^{*}}$ \\ ${ }^{1}$ Department of Neurology, the First Affiliated Hospital of Dalian Medical University, Dalian, Liaoning, China \\ ${ }^{2}$ Dalian Liguang Rehabilitation Hospital
} Analysis

*Corresponding Author: Ming-Ou Lu, Department of Neurology, the First Affiliated Hospital of Dalian Medical University, Dalian, Liaoning, China

Received date: February 24, 2021; Accepted date: April 19, 2021; Published date: April 26, 2021

Citation: Duo-Duo Fan., Zhe Wang., Wen-Song Mu., Ming-Ou Lu., (2021) A Case Report of Classical eight-and-a-Half Syndrome and Etiology Analysis. J. Neuroscience and Neurological Surgery. 9(2); DOI:10.31579/2578-8868/162

Copyright: $\odot 2021$ Ming-Ou Lu, This is an open-access article distributed under the terms of The Creative Commons Attribution License, which permits unrestricted use, distribution, and reproduction in any medium, provided the original author and source are credited

\begin{abstract}
:
Eight-and-a-half syndrome is one-and-a-half syndrome plus ipsilateral seventh (facial) cranial nerve palsy, which was first reported by Eggenberger in 1998. This syndrome is caused by a lesion (most often vascular or demyelinating) in the dorsal tegmentum of the caudal pons. It involves the para pontine reticular formation and the medial longitudinal fasciculus, as well as the nucleus and the fasciculus of the facial nerve. We describe a case of a 56-year-old man presented with eight-and-a-half syndrome. We also discuss the vascular pathology and etiology of this syndrome through the literature review. Most eight-and-a-half syndrome cases are caused by occlusions of the perforating branches of the pons. The pathology can be transparent lipid-like changes at the perforator arteries. It can also be thrombosis at the orifice of the main or perforator arteries. BAH could be a very important factor in perforator artery infarction at the pontine. The etiology is closely related to hyperglycemia. An effective intervention is to avoid bad habits and control the hyperglycemia.
\end{abstract}

Keywords: eight-and-a-half syndrome; perforator artery; transparent lipid-like changes

\section{Introduction}

Eight-and-a-half syndrome is one-and-a-half syndrome plus ipsilateral seventh (facial) cranial nerve palsy, which was first reported by Eggenberger in 1998 [1]. The lesion of this syndrome is at the dorsal tegmentum of the caudal pons involving the para pontine reticular formation and the medial longitudinal fasciculus, as well as the nucleus and the facial nerve [2]. Xia et al. categorizes the spectrum of eight-anda-half syndrome into three types, namely classic eight-and-a-half syndrome, eight-and-a-half syndrome variants and eight-and-a-half plus syndrome. Eight-and-a-half plus syndrome includes nine / thirteen-and-ahalf / fifteen-and-a-half syndrome [3]. Typical classic eight-and-a-half syndrome shows a combination of partial horizontal gaze palsy associated with ipsilateral facial lower motor neuron paresis [4]. Due to its rarity, the clinical course and the pathogenesis of this specific disease are not well established. The common causes are pons infarction, infection, multiple sclerosis, tumor,etc, among which infarctions are most frequent. Several cases of this syndrome by infarction have been reported, but the etiology was not well elaborated. We report a classic case of eight-and-a-half syndrome in which the brain MRI (magnetic resonance imaging) revealed a pontine tegmentum infarction. We also discuss the vascular pathology and etiology of eight-and-a-half syndrome by literature review.

\section{Case report:}

The patient, a 56-year-old male, was admitted to our hospital with the complaint of "sudden dizziness accompanied by blurred vision for one day". His medical history includes hypertension for 5 years (up to 200/120 $\mathrm{mmHg}$ ), coronary heart disease for 6 years, possible but unconfirmed history of diabetes, smoking (20 cigarettes a day) and alcohol consumption (white spirit $250 \mathrm{~g}$ a day) for more than 30 years. Physical examination showed BP of $140 / 75 \mathrm{mmHg}$, restricted adduction of the left eye, nystagmus when gazing left, restricted abduction, adduction of the right eye and the right eyeball was fixed in the middle. Up- and downward movement of both eyes was normal (Figure 1a). His right frontal wrinkles disappeared, right palpebral fissure was larger and right nasolabial folds were shallower (Figure 1b).

Limb strengths were normal. Bilateral Babinski signs were negative. The fasting blood glucose and the glycated hemoglobin were elevated. There was also hyperlipidemia. Head MRI and DWI (diffusion weighted imaging) revealed an acute infarction in the right side of the dorsal pons. MRA (magnetic resonance angiography) showed that the left vertebral artery and internal carotid artery were slightly narrower (Figure 2). Antiplatelet aggregation, blood pressure regulation and lipid regulation were given to stabilize plaque, improve circulation and protect neurons. Symptoms improved after 7 days of treatments. After a month of 
rehabilitation, the symptoms improved significantly (Figure 1c). The patient was basically normal on the 5th month follow-up. (Figure 1d).
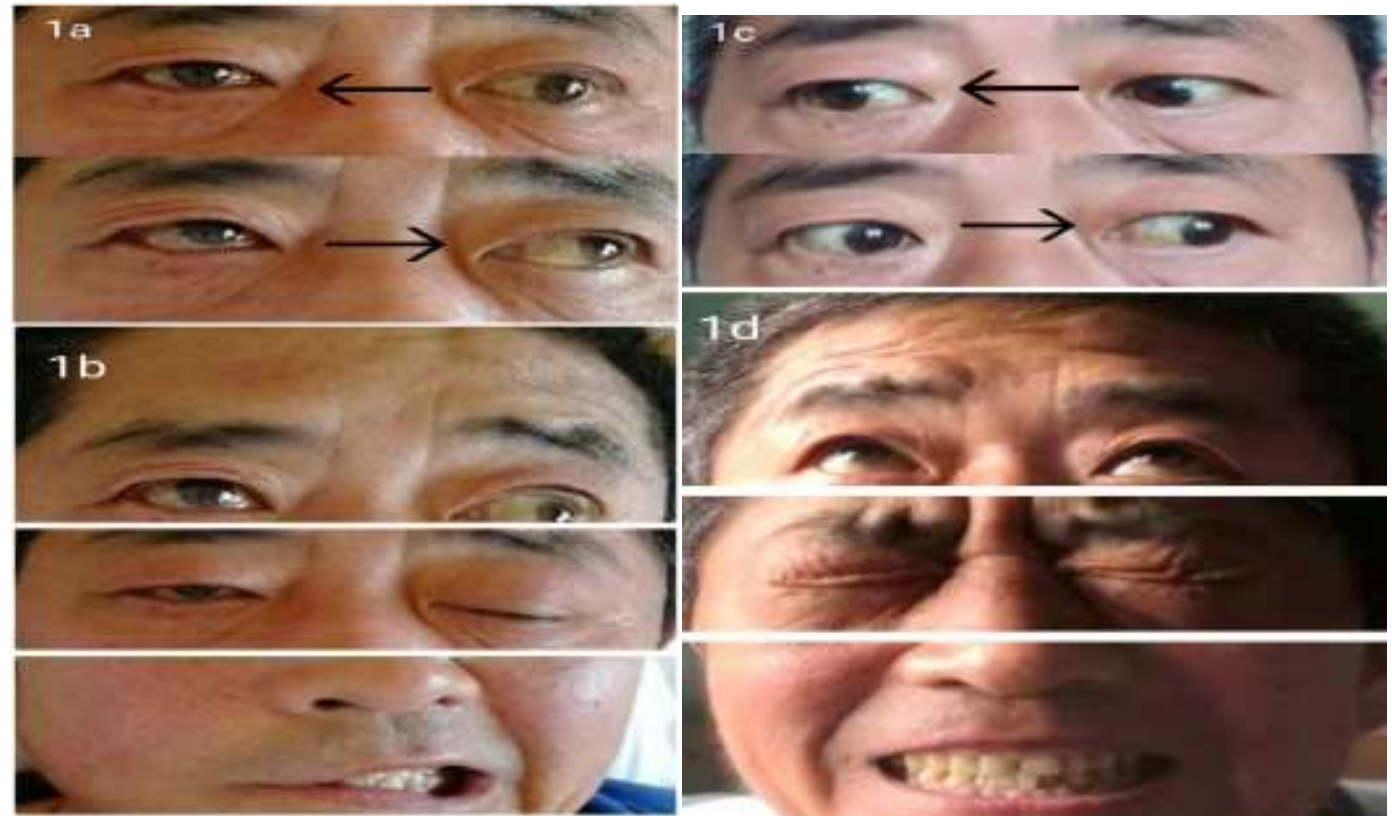

Figure 1: $a$. On the third day of onset, the adduction movement of the left eye was restricted, the adduction and abduction movements of the right eye were restricted and eyeball was fix in the middle, the upward and downward movements of both eyes are generally normal; $b$. Peripheral facial paralysis on the third day of onset; $\boldsymbol{c}$. On first month follow-up, eye movements abnormality improved significantly; $d$. Peripheral facial paralysis disappeared on the fifth month follow-up.

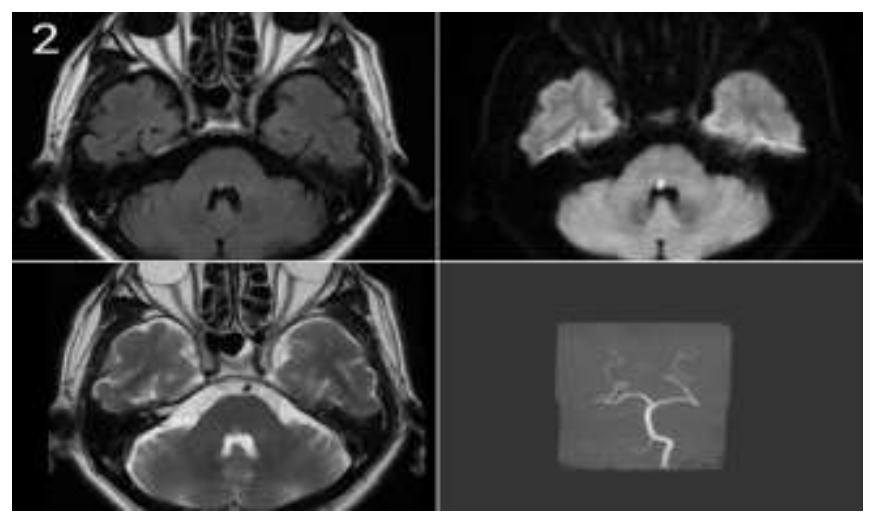

Figure 2: The brain MRI、DWI and MRA of the patient.

\section{Discussion:}

Our patient showed a complete right (ipsilateral) horizontal gaze paresis, partial gaze paresis of the left eye (spared abduction), and right peripheral facial nerve paresis. MRI revealed a right caudal pontine infarction involving the para pontine reticular formation, medial longitudinal fasciculus and VII nerve nucleus. These conformed to the classic eightand-a-half syndrome.

Studies have shown that most pontine infarctions are caused by transparent lipid-like changes in perforator arteries [5.6]. The pontine perforator artery originates from the basilar artery, and mainly consists of the paramedian branches, the pontine long- and short-circumferential branches. The collateral circulation of these arteries is very poor because they are terminal arteries with small diameters. Also, these arteries are hardly connected to the anterior or posterior cerebral artery [7], so the occlusions of these perforator arteries have almost no compensatory mechanisms.

There are two types of pathological changes in the infarction of perforator arteries: 1) atherosclerosis resulting in thrombosis at the orifice of perforator artery or main artery 2) transparent lipid-like changes leading to arterial wall occlusion. Their clinical treatments and prognosis are different [8]. Based on Figure 2, we suppose that the lesion in our patient is caused by transparent lipid-like changes in the distal part of the long circumferential branches.

A clinical controlled study of 120 patients showed that the etiological factors of most atherosclerotic cerebral infarctions with large arteries were due to alcohol consumption, smoking and abnormal lipid metabolism. However, perforator artery infarction is more related to diabetes mellitus [9]. This is verified by many reported eight-and-a-half 
syndromes [2.3.4.10.]. Therefore, strict control of blood glucose is an effective preventive measure of perforator artery infarction.

A study of 328 patients with cerebral infarction showed that incidence of basilar artery hypoplasia (BAH) with perforator artery infarction is $8.2 \%$, and it is even higher in the posterior circulation part, especially in pontine perforator artery infarctions [11]. This suggests that BAH may be one of the important vascular causes of pontine perforator artery infarction. This is consistent with the conclusion that congenital arterial hypoplasia usually results in small scattered infarctions [12]. Normally the diameter of the basilar vessel in BAH is evenly smaller (below $2 \mathrm{~mm}$ at pontine). The diameter of the basilar artery (pontine level) in our patient is about $2.8 \mathrm{~mm}$, which can exclude BAH.

\section{Conclusion}

Most eight-and-a-half syndromes are caused by occlusions of the perforating branches of pons. The pathology can be transparent lipid-like changes of the perforator arteries, or thrombosis at the orifice of the main / perforator arteries. BAH could be a very important factor in perforator artery infarction at the pontine. The etiology is closely related to hyperglycemia. An effective intervention is to avoid bad habits and control the hyperglycemia.

\section{References:}

1.Eggenberger E. Eight-and-a-half syndrome: one-and-a-half syndrome plus cranial nerve VII pals. J Neuroophthalmol. 1998; 18(2): 114- 116.

2.Bocos-Portillo J, Ruiz Ojeda J, Gomez-Beldrrain M, et al. Eightand-a-Half Syndrome, Jama Neurol. 2015; 72(7): 830.

3.Xia NG, Chen YY, Li J, et al. Eight-and-a-half syndrome caused by a pontine hemorrhage: a case report and review of the literature. Intl. J. Neurosci. 2018; 128(8):746-750.
4. Karkar P, Brown Z, Banerjee S. Eight-and-a-half syndrome: an unusual presentation of brainstem infarction. Q J Med. 2013; 106:273-276.

5. Chen $\mathrm{H}$, Wang $\mathrm{Y}$, Hua $\mathrm{H}$, et al. Unilateral isolated pontine infarction involving the surface and interior of the pons. Chinese $\mathrm{J}$ Neurol. and Psy. Dis. 2011; 37(5): 280- 284.

6.Kobayashi J, Ohara T, Minematsu K, et al. Etiological mechanisms of isolated pontine infarcts based on arterial territory involvement. J. Neurol. Sci. 2014; 339: 113- 117.

7.Zhu L, Hoffmann A, Wintermark M, et al. Do microemboli reach the brain penetrate arteries? Surg Res. 2012; 176(2): 679- 683.

8. Kaptoge S, Di Angelantonio E, Lowe G, et al. C-reactive protein concentration and risk of coronary heart disease, stroke, and mortality: an individual participant meta-analysis. Lancet. 2010; 375(9709): 132- 140.

9.Liu Y. Comparison of clinical characteristics between atherosclerotic cerebral infarction and perforator cerebral infarction. China \&Foreign Medical Treatment. 2016; 16:57- 58.

10. Ahmed HA, Al Assaf OY, Alzarooni HM, et al. Clinical findings of eight-and-a-half syndrome. Oxford Medical Case Reports. 2020; 10, 357-358.

11. Zhang DP. Study on the Role of Dysplasia of Basilar Artery in Patients with Pontine Perforator Artery Infarction. Chinese J Cardiocerebrovascular Dis for the Aged. 2012; 14(10): 1036-1038.

12. Park JH, Roh JK, Kwon HM. Ischemic stroke patterns and hemodynamic features in patients with small vertebrobasilar artery. J Neurol Sci. 2009; 287: 227- 235.

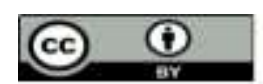

This work is licensed under Creative Commons Attribution 4.0 License
To Submit Your Article Click Here: Submit Manuscript

DOI:10.31579/2578-8868/162
Ready to submit your research? Choose Auctores and benefit from:

* fast, convenient online submission

* rigorous peer review by experienced research in your field

* rapid publication on acceptance

* authors retain copyrights

* unique DOI for all articles

* immediate, unrestricted online access

At Auctores, research is always in progress.

Learn more www.auctoresonline.org/journals/neuroscience-andneurological-surgery 